\title{
2D-Drop model applied to the calculation of step formation energies on a (111) substrate.
}

\author{
M.I. Rojas, G. E. Amilibia, M.G. Del Pópolo and E.P.M. Leiva* \\ INFIQC. Unidad de Matemática y Física \\ Fac. Cs. Químicas, Universidad Nacional de Córdoba \\ Ciudad Universitaria, 5000 \\ Córdoba, Argentina.
}

November 15, 2018

\begin{abstract}
A model is presented for obtaining the step formation energy for metallic islands on (111) surfaces from Monte Carlo simulations. This model is applied to homo $(\mathrm{Cu} / \mathrm{Cu}(111), \mathrm{Ag} / \mathrm{Ag}(111))$ and heteroepitaxy $(\mathrm{Ag} / \mathrm{Pt}(111))$ systems. The embedded atom method is used to represent the interaction between the particles of the system, but any other type of potential could be used as well. The formulation can also be employed to consider the case of other single crystal surfaces, since the higher barriers for atom motion on other surfaces are not a hindrance for the simulation scheme proposed.

Keywords: Step formation energy, Monte Carlo simulation, submonolayers.
\end{abstract}

\section{Introduction}

The formation of metal islands on a metal surface represents an important stage in the growth of a phase in the case of homoepitaxy, or the appearance of a new one in the case of heteroepitaxy. In any case, this topic has interested

*Corresponding author. Fax 54-351-4334189; e-mail: eleiva@fcq.unc.edu.ar 
whole generations of theoreticians and experimentalists, and is currently the subject of intense research. Just trying to cite relevant work in this field would require by itself a whole extensive review, so we just mention briefly the work which may be connected directly to the present letter.

Concerning homoatomic systems, recent experimental work has allowed the accurate determination of the equilibrium shape of two dimensional metal islands on different single crystal surfaces[1]. The analysis by the inverse Wulff construction allowed the determination of the ratio of the free energies per step length of the A- and B- type steps on $\mathrm{Cu}(111)$ and $\mathrm{Ag}(111)$ surfaces, as well as the angle dependence of the step free energy for $\mathrm{Cu}(100), \mathrm{Cu}(111)$ and $\mathrm{Ag}(111)$. The analysis of the experimental data using Ising models worked quite well for the (111) surfaces but were not helpful in general for the (100) surfaces. On the other hand, an alternative method for determining the kink energy from Arrhenius plots was developed, which did not rely on a specific model for the interactions between the atoms but on thermodynamic arguments. An important quantity also emerging from these studies was the step energy per atom, which was obtained for straight and $100 \%$ kinked steps, thus yielding a detailed picture of the energetics of these systems.

In the case of heteroatomic systems, the interatomic interactions and the adsorbate/substrate misfit determine the structure and the growth mechanism of the monolayer and subsequent adlayers[2]. Although the experimental data on these systems is extensive, information concerning the step formation energy is scarce. This quantity has been estimated from electrochemical measurements by Xia et al [3] for the system $\mathrm{Cu} / \mathrm{Au}(111)$, finding a value of $0.4-0.5 \mathrm{eV} /$ (step atom). As we shall see below, in the case of deposition of $\mathrm{Ag}$ on $\mathrm{Pt}(111)$ [4] a rough estimation of this quantity can also be made from the temperature at which the fragmentation of islands occurs.

It is the purpose of the present work to formulate a model for the estimation of step formation energies from Monte Carlo simulations, and perform calculations for some typical systems. Our model includes the natural relaxation of the system due to temperature effects, which coupled to the misfit between substrate and adsorbate may produce some singular effects in certain systems. Due to the simplified potential employed, we do not expect to make accurate quantitative predictions, but we think the present results will motivate further research in the field. The systems studied were $\mathrm{Cu} / \mathrm{Cu}(111)$, $\mathrm{Ag} / \mathrm{Ag}(111)$ and $\mathrm{Ag} / \operatorname{Pt}(111)$. 


\section{The Drop model}

In the present calculations we focus on flat islands that are only one atom thick. Both the energy of adsorption per atom in the bulk of the island and edge may be of interest. In order to separate the two contributions, we have adapted the drop model [5] that is often used for the calculation of surface energies to the two-dimensional situation. This model permits to obtain the binding energy of the atoms in the monolayer $u_{s}$ and the binding energy of the atoms at the border of the island $u_{b}$ from the adsorption energy per atom $u_{t}$, the number of border atoms $N_{b}$ and the number of inner atoms $N_{s}$ that can be obtained for the Monte Carlo simulation.

Let $U_{T}$ be the excess of energy of a system composed of an island adsorbed on a substrate surface $M$ :

$$
U_{T}=\left(U_{A / M}-U_{M}\right)
$$

where $U_{A / M}$ is the energy of the adsorbed-substrate system and $U_{M}$ is the energy of the substrate without adsorbate.

If we consider the adsorbed submonolayer like a 2-D drop, $U_{T}$ can be expressed in terms of the edge atoms $N_{b}$ and the number of atoms which make up the surface $N_{s}$ as:

$$
U_{T}=u_{s} N_{s}+u_{b} N_{b}
$$

Let $N$ be the total number of atoms in the island:

$$
N=N_{s}+N_{b}
$$

If we replace $N_{s}$ from this equation into equation 1, we obtain:

$$
U_{T}=u_{s} N+\left(u_{b}-u_{s}\right) N_{b}
$$

And dividing by $N$ we get:

$$
u_{t}=\frac{U_{T}}{N}=u_{s}+\left(u_{b}-u_{s}\right) \frac{N_{b}}{N}
$$

Thus, a linear relationship is expected between the average values $\left\langle u_{t}\right\rangle$ and $\frac{\left\langle N_{b}>\right.}{N}$ for simulations employing islands of different sizes. The $u_{s}$ and $u_{b}$ were obtained using least squares to calculate the ordinate and slope of the straight line respectively. 
The ordinate $u_{s}$, corresponding to the limit $\frac{N_{b}}{N} \rightarrow 0$ should approach the binding energy of a monolayer. The slope $\left(u_{b}-u_{s}\right)$ is the border formation energy per atom. This should always be a positive quantity and its amount indicates the energy change required to bring an atom from the surface to the edge of the island.

\section{Model and Computations}

The substrate surface was assumed to be smooth without considering steps or reconstruction. The substrate employed in Monte Carlo simulations was a 4-layer slab with 480 atoms per layer to represent (111) crystalline surface. The atoms in the first and the second planes of the substrate located within a circular area containing ca. 250 atoms were allowed to vary the positions during the simulation. The remaining substrate atoms were fixed to their bulk equilibrium configuration to emulate the presence of a semi-infinite crystal. On this substrate, submonolayers of an initially circular shape containing between 12-150 atoms were adsorbed to emulate islands of different sizes.

The potentials employed were given by the embedded atom method (EAM) which takes into account many body interactions characteristic of the metallic systems [6], [7]. The total energy is expressed in terms of two contributions:

$$
E_{\text {tot }}=\sum_{i=1}^{N} F_{i}\left(\rho_{h, i}\right)+\frac{1}{2} \sum_{i} \sum_{j \neq i} \phi_{i j}\left(r_{i j}\right)
$$

where $\rho_{h, i}$ is the host electronic density at $i$ atom position, $F_{i}(\rho)$ is the embedding energy and $\phi_{i j}\left(r_{i j}\right)$ is the core-core pair repulsion between $i$ and $j$ atoms separated at a $r_{i j}$ distance. In all cases we used the parametrization of Foiles et al [7].

All the Monte Carlo simulations were performed in the (NVT) ensemble at $300 \mathrm{~K}$. We considered 5000 equilibration steps followed by 15000 production steps.

In order to let the adatoms overcome high energy barriers, like those for the displacement on the surface or the detachment from a cluster, we allowed these adatoms to perform long jumps, with displacements $\Delta r$ :

$$
\Delta r=n_{1} \overrightarrow{s_{1}}+n_{2} \overrightarrow{s_{2}}
$$


where $n_{1}$ and $n_{2}$ are integers and $\overrightarrow{s_{1}}$ and $\overrightarrow{s_{2}}$ are the primitive vectors of a two-dimensional Bravais lattice. These "long" jumps are important to get a proper equilibration of the system. "Short" jumps, as usually employed in Monte Carlo simulations where the positions of the particles are varied continuously, are also present in our studies to describe the vibrational motion of the adsorbate atoms in the neighborhood of their equilibrium positions. This simulation method was employed in a previous work [8] to study $\mathrm{Pd}$ submonolayer growth on different monocrystalline surfaces, yielding results in reasonable agreement with experimental data of literature.

\section{Results and Discussion}

The equilibrium shapes of two dimensional islands have been the subject of extensive research in both the experimental and the theoretical fields. As mentioned above, a recent work of Giesen et al has analyzed this problem in detail[1]. While our current computer capabilities do not allow the performance of simulations able to predict the shapes of island with sizes in the order of tenths of nanometers, we shall tackle the problem of predicting step formation energies.

We performed simulations for the homoatomic systems $\mathrm{Cu} / \mathrm{Cu}(111)$ and $\mathrm{Ag} / \mathrm{Ag}(111)$ and for the heteroatomic $\mathrm{Ag} / \mathrm{Pt}(111)$ system for a series of islands of different sizes. The values of $\left\langle u_{t}\right\rangle$ and $\frac{\langle N b\rangle}{N}$ were obtained from the simulation production steps for the different submonolayer sets and are plotted in Figure 1. We also show there the $\left\langle u_{t}\right\rangle$ values obtained from a simulation with a monolayer. We have plotted these monolayer energies assigning them the value $\frac{\langle N b\rangle}{N}=0$. For the larger $N$ employed in our simulations, we observe a linear behavior for the three systems studied. A least square fitting in the range $0.2<\frac{\langle N b\rangle}{N}<0.4$ using eqn. (2) delivered the $u_{s}$ and $u_{b}$ reported in Table 1 . This set corresponds to the larger islands simulated. For $\mathrm{Cu} / \mathrm{Cu}(111)$ and $\mathrm{Ag} / \mathrm{Ag}(111)$ the step formation energies $u_{b}-u_{s}$ are in the order of $0.2-0.3 \mathrm{eV}$, which are close to the values found by Giesen et al. [1]. In the case of the $\mathrm{Ag} / \mathrm{Pt}(111)$ system, an unusually low step formation energy is found. This system presents an important positive misfit $(4.3 \%)$ which generates a strong compressive stress in the pseudomorphic $(1 \times 1)$ monolayer [9]. Röder et al [4 have shown that when islands are grown at low temperatures and subsequently annealed, a remarkable morphology transition is found around 300-350 K. At these temperatures, island 
fragmentation occurs via edge roughening and kink evaporation, so that at $400 \mathrm{~K}$ all large Ag islands are eventually disintegrated. Generally speaking, island disintegration should be expected to take place when the step formation energy is of the order of kT. Our estimation of step formation energy of ca. $0.085 \mathrm{eV}$, small compared with those of other systems, delivers a disintegration temperature $\left(990{ }^{\circ} \mathrm{K}\right)$ which results far above the experimental value. While this disagreement could be roughly attributed to the approximate nature of the potential employed, close observation of figure 1 shows that the linear extrapolation of the $\left\langle u_{t}\right\rangle$ vs $\frac{\langle N b\rangle}{N}$ curve to $\frac{\langle N b\rangle}{N} \rightarrow 0$ shows the strongest deviation with respect to the $\left\langle u_{t}\right\rangle$ of the monolayer for the $\mathrm{Ag} / \mathrm{Pt}(111)$ system. Thus, the question arises if some of the approximations involved in the present model are responding bad for this system. In this sense, the extrapolation to $\frac{\langle N b>}{N} \rightarrow 0$ to obtain the ordinate $u_{s}$ from equation (2) implies the assumption that the island is roughly composed of two sorts of atoms, say "border" and "inner " atoms. In order to learn how fast the atoms inside the island approach the behavior of atoms in a monolayer ( "inner " atoms), we have plotted in figure 2 the distance between nearest neighbors at the center of the monolayer as a function of the total number of atoms in the island. In the case of the system $\mathrm{Ag} / \mathrm{Ag}(111)$, we see that for relatively small islands the atoms at the center already reflect the behavior of the monolayer. In the case of the $\mathrm{Cu} / \mathrm{Cu}(111)$ system, the approach to the monolayer behavior is more sluggish, but is practically reached for the large island in our simulation. On the other hand, we see that in the case of the $\mathrm{Ag} / \mathrm{Pt}(111)$ system the behavior of the inner atoms is far from that of $\mathrm{Ag}$ atoms in a (1x1) monolayer on $\mathrm{Pt}(111)$. These results indicate that small $\mathrm{Ag}$ islands on $\mathrm{Pt}(111)$ are considerably expanded with respect to the $(1 \mathrm{x} 1)$ monolayer, becoming more and more compressed with growing island size, probably reaching finally a $(1 \mathrm{x} 1)$ structure with the substrate for sizes that are beyond our simulation conditions. In this way, the binding energy of the atoms in the monolayer is not accurately estimated from the extrapolation in figure 1 . On the other hand, the $u_{s}$ values can be properly obtained from a simulation employing a (1x1) adsorbed monolayer, and the linear fitting of the points in figure 1 can be made by involving $u_{b}$ as unique free parameter. Table 2 shows $u_{s}$ values stemming from monolayer simulations, as well as the $u_{b}$ obtained from linear fittings taking $u_{s}$ from these monolayer simulations. The corresponding step formation energies $u_{b}-u_{s}$ are also reported there. Comparison with Table 1 indicate that although the extrapolated $u_{s}$ values were reasonable, important changes occur in the step formation energy, es- 
pecially in the case of $\mathrm{Ag} / \mathrm{Pt}(111)$, where $u_{b}-u_{s}$ has strongly decreased.

A last point that we have checked is the influence of relaxation in the calculated adsorption and step formation energies. In Table 3 we show the quantities obtained from static calculations, where the island and surface atoms are not allowed to relax. As in the previous case, the most drastic differences are found in the case of the step formation energy of the system $\mathrm{Ag} / \operatorname{Pt}(111)$.

For all the island sizes of $\mathrm{Ag} / \mathrm{Pt}(111)$ considered in this work, we found an outwards relaxation of the edge atoms of the islands, which confirms the statement of Bromann et al [10] concerning the fact that compressive stress can be relieved by this way. However, we did not find pseudomorphic growth in our islands, but rather expanded structures which become progressively compressed as the islands increase their size. Simulations using periodic boundary conditions showed that the $(1 \times 1)$ structure was stable under our simulation conditions. Thus, the picture that emerges from the present calculations is that the increased size of the islands leads to progressively compressed structures of $\mathrm{Ag}$ on $\mathrm{Pt}(111)$ until they reach the $(1 \times 1)$ structure of the monolayer. The actual behavior of the experimental system is richer, with the appearance of partial dislocations, yielding domain walls between fcc and hcp stacking [10]. In this respect, simulations with a much larger number of atoms are desirable in order to investigate if the EAM potential is able to reproduce such subtle features.

In contrast to the $\mathrm{Ag} / \mathrm{Pt}(111)$ system, the other systems present inwards relaxation at the edge, following the natural expectation from bond-order conservation analysis, atoms at the edge are less coordinated, and they increase the strength of the binding with each other.

We can summarize the present results by stating that the model we have presented permits to obtain the step formation energy for metallic islands on (111) surfaces in a straightforward way for homo and heteroepitaxy systems from Monte Carlo simulations. We have used the embedded atom method to represent the interaction between the particles of the system, but any other type of potential could be used as well. The model can also be used for other single crystal surfaces, since the higher barriers for atom motion on other surfaces are not a hindrance for the simulation scheme proposed. 


\section{Acknowledgments}

This work was supported by PIP and PEI 86/98 CONICET, Program BID 802/OC-AR PICT 06-04505, CONICOR, Secyt UNC, Argentina. We also wish to thank M.I. Baskes for providing the tables with the potential functions. Language assistance from P. Falcon is also acknowledged. 


\section{Tables}

\begin{tabular}{|c|c|c|c|}
\hline System & $u_{s}[\mathrm{eV}]$ & $u_{b}[\mathrm{eV}]$ & $u_{b}-u_{s}[\mathrm{eV}]$ \\
\hline \hline $\mathrm{Cu} / \mathrm{Cu}(111)$ & $-3.509 \pm 0.001$ & $-3.194 \pm 0.004$ & $0.315 \pm 0.003$ \\
\hline $\mathrm{Ag} / \mathrm{Ag}(111)$ & $-2.817 \pm 0.001$ & $-2.583 \pm 0.004$ & $0.234 \pm 0.003$ \\
\hline $\mathrm{Ag} / \mathrm{Pt}(111)$ & $-3.167 \pm 0.002$ & $-2.997 \pm 0.008$ & $0.085 \pm 0.006$ \\
\hline
\end{tabular}

Table 1: Binding energy per atoms in the monolayer $u_{s}$, binding energy per atom at the edge of the island $u_{b}$ and step formation energy per atom $u_{b}-u_{s}$ for different systems. These values were obtained from linear fitting of the points in figure 1 in the range $0.2<\frac{\leq N b>}{N}<0.4$. The parameters fitted were $u_{s}$ and $u_{b}-u_{s}$ according to equation (2). The Monte Carlo/EAM simulations were performed at $300 \mathrm{~K}$.

\begin{tabular}{|c|c|c|c|}
\hline System & $u_{s}[\mathrm{eV}]$ & $u_{b}[\mathrm{eV}]$ & $u_{b}-u_{s}[\mathrm{eV}]$ \\
\hline \hline $\mathrm{Cu} / \mathrm{Cu}(111)$ & -3.498 & $-3.216 \pm 0.002$ & $0.283 \pm 0.002$ \\
\hline $\mathrm{Ag} / \mathrm{Ag}(111)$ & -2.808 & $-2.600 \pm 0.001$ & $0.208 \pm 0.001$ \\
\hline $\mathrm{Ag} / \mathrm{Pt}(111)$ & -3.150 & $-3.118 \pm 0.002$ & $0.032 \pm 0.002$ \\
\hline
\end{tabular}

Table 2: Binding energy per atom in the monolayer $u_{s}$, binding energy per atom at the edge of the island $u_{b}$ and step formation energy per atom $u_{b}-u_{s}$ for different systems. The $u_{s}$ were obtained from monolayer simulations. The $u_{b}$ values were obtained from linear fitting of the points in figure 1 in the range $0.2<\frac{\langle N b\rangle}{N}<0.4$ according to equation (2). The Monte Carlo/EAM simulations were performed at $300 \mathrm{~K}$. 


\begin{tabular}{|c|c|c|c|}
\hline System & $u_{s}[\mathrm{eV}]$ & $u_{b}[\mathrm{eV}]$ & $u_{b}-u_{s}[\mathrm{eV}]$ \\
\hline \hline $\mathrm{Cu} / \mathrm{Cu}(111)$ & $-3.555 \pm 0.003$ & $-3.21 \pm 0.01$ & $0.350 \pm 0.007$ \\
\hline $\mathrm{Ag} / \mathrm{Ag}(111)$ & $-2.861 \pm 0.002$ & $-2.597 \pm 0.008$ & $0.264 \pm 0.006$ \\
\hline $\mathrm{Ag} / \mathrm{Pt}(111)$ & $-3.164 \pm 0.002$ & $-3.001 \pm 0.003$ & $0.163 \pm 0.001$ \\
\hline
\end{tabular}

Table 3: Binding energy per atom in the monolayer $u_{s}$, binding energy per atom at the edge of the island $u_{b}$ and step formation energy per atom $u_{b}-u_{s}$ for different systems, as obtained from static EAM calculations. These values arise from a linear fitting of the adsorption energy per atom in the range $0.2<\frac{\langle N b>}{N}<0.4$. The parameters fitted were $u_{s}$ and $u_{b}-u_{s}$ according to equation (2). 


\section{References}

[1] M. Giesen, C. Steimer and H. Ibach, Surface Science 471(2001)80.

[2] W.J. Lorenz, G. Staikov, Surf. Sci. 335 (1995) 32-43.

[3] X.H. Xia, R. Schuster, V. Kirchner and G. Ertl, J. Electroanal Chem. 461(1999)102.

[4] H. Röder, H. Brune and K. Kern, Phys. Rev. Lett. 73(1994)2143.

[5] L. Szybisz, Physica A 283 (2000) 193.

[6] M.S. Daw and M.I. Baskes, Phys. Rev. B 50 (1983) 1285.

[7] S.M. Foiles, M.I. Baskes and M.S. Daw, Phys. Rev. B 33 (1986) 7983.

[8] M.I. Rojas M.G. Del Pópolo and E.P.M. Leiva, Langmuir 16 (24) (2000) 9539.

[9] H. Ibach, Surf. Sci. Rep. 29 (1997) 193.

[10] K. Bromann, H. Brune, M. Giovannini, K. Kern, Surf. Sci. Lett. 388 (1997) L1107. 


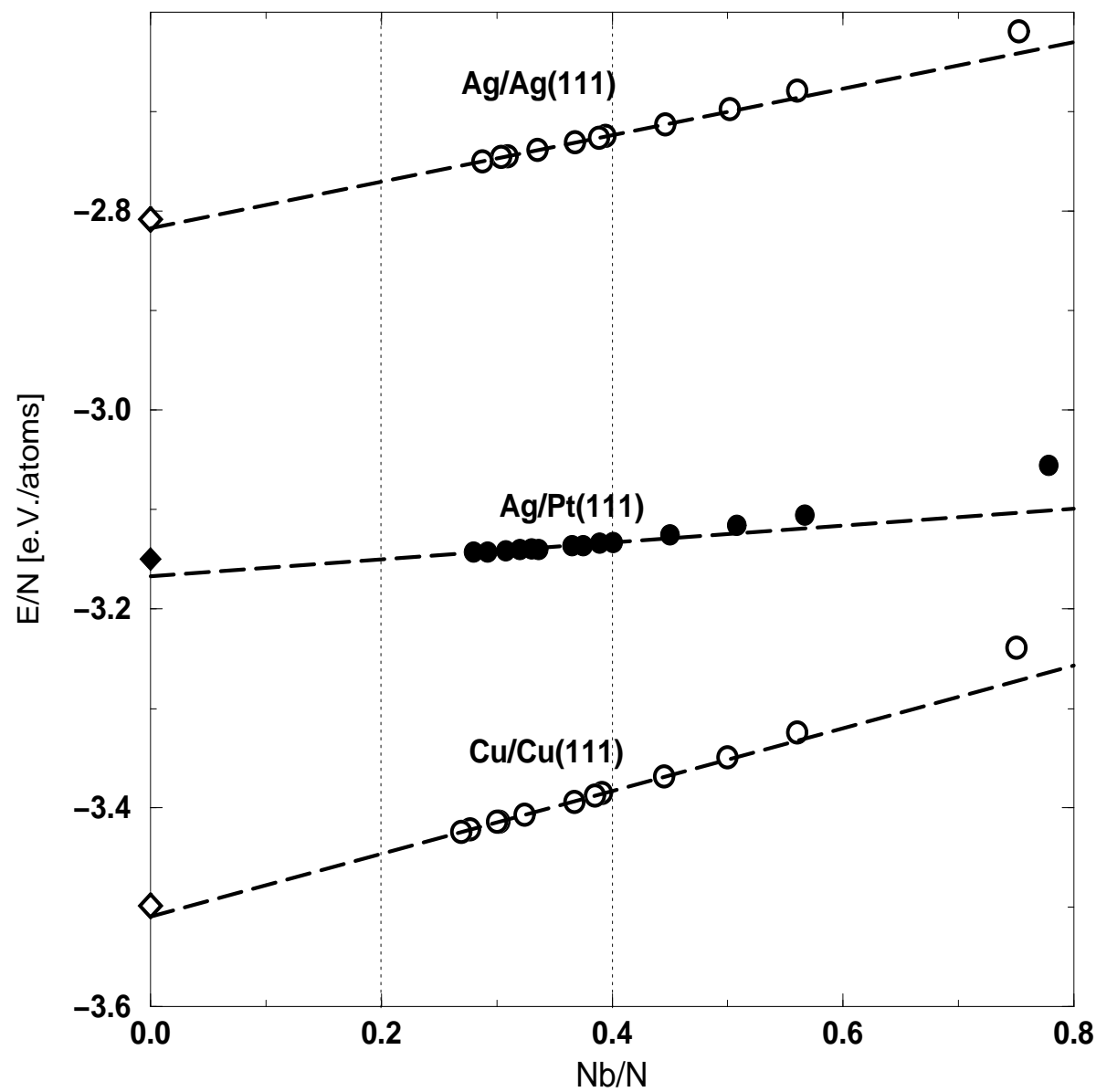

Figure 1: Adsorption energy per atom $\left\langle u_{t}>\right.$ vs fraction of border atoms $\frac{\langle N b>}{N}$ curve for different adsorbate(island)/substrate(111) systems. The points at $\frac{\langle N b>}{N}=0$ correspond to monolayer simulations. 


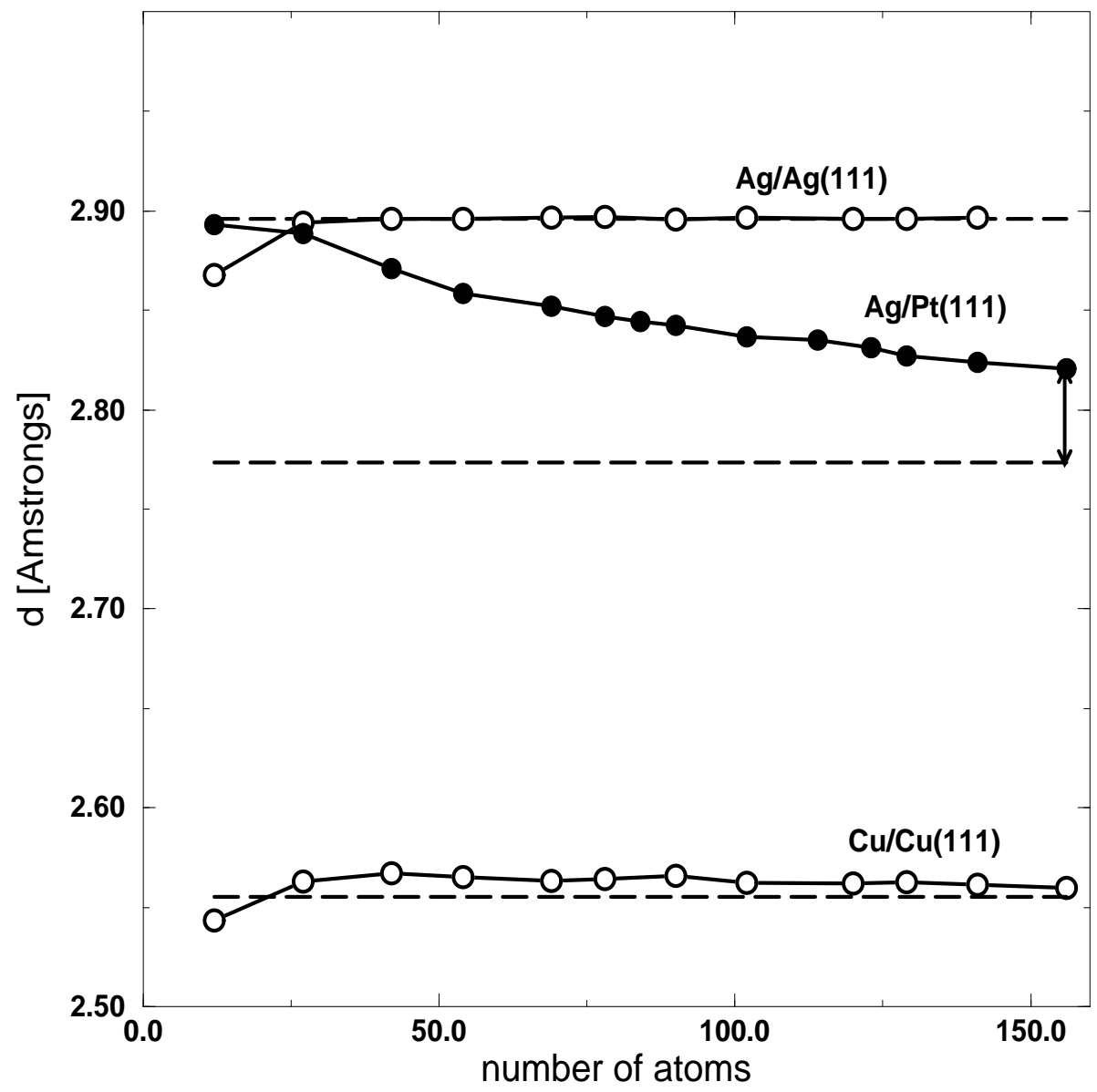

Figure 2: Nearest neighbor distance at the center of the island as a function of the total number of island atoms for the systems considered in this work. 